\title{
Addressing the Need, Ethical Decision Making in Disasters, Who Comes First?
}

Claritza L. Rios ${ }^{1}$, Michael Redlener ${ }^{2}$, Eric Cioe $^{1}$, Patricia M. Roblin ${ }^{1}$, Stephan Kohlhoff ${ }^{1}$, Stephan Rinnert ${ }^{3}$, William Lang ${ }^{4}$, Kathleen Powderly ${ }^{5}$ and Bonnie Arquilla ${ }^{1}$

1. Department of Emergency Medicine, SUNY Downstate Medical Center, Brooklyn, NY 11203, United States

2. Fire Department City of New York, Fort Totten, NY 11359, United States

3. Department of Emergency Medicine ,Kings County Hospital Center, Brooklyn, NY 11203, United States

4. New York City Department of Health and Mental Hygiene, New York, NY 11203, United States

5.The John Conley Division of Medical Ethics and Humanities, SUNY Downstate Medical Center, Brooklyn, NY 11203, United States

\begin{abstract}
Disasters exhaust resources, complicate care, and force a level of decision-making that is outside the usual framework of medical care providers. A group of experts was convened to discuss and explore the ethical decision-making that takes place during a disaster. 37 professionals from the fields of emergency medicine, emergency planning, emergency services, clinical ethics, public health, palliative care and various clinical disciplines were tasked with ethical decision-making in a disaster. They were divided into three breakout groups and presented with a detailed drill scenario involving mass casualties following an explosion and partial building collapse with several victims presenting simultaneously in the Emergency Department with similar severe injuries and were tasked with deciding which patient would be treated first. The groups considered various principles in establishing the triage approach for the scenario patients which included "social value", and first come first served but ultimately, they triaged the patients based primarily on their clinical presentations and likely prognosis, followed by age and professional affiliations in the case of similar medical conditions and had similar outcomes. The groups also agreed that the presence of palliative care in the setting of disaster, as well as, including community leaders in disaster planning would be of critical assistance. A senior physician should be assigned as a primary decision maker and leader in a mass casualty incident. Participation of a palliative care teams during crisis and disaster situations would expand the options of care and help the family feel more comfortable with difficult decisions regarding limiting care for their loved ones. Community involvement prior to disaster was strongly recommended to help communication and trust between community and practitioners and to also assign point people.
\end{abstract}

Key words: Emergency preparedness, ethics, ethical decision making.

\section{Introduction}

Disasters exhaust resources, complicate care, and force a level of decision-making that is outside the usual framework of medical care providers. One only has to look toward recent events-Hurricane Katrina in New Orleans, Louisiana; the earthquake in Haiti; the earthquake and tsunami in Japan [1-3], to appreciate the complexities of such disasters. During disasters, physicians are often called upon to make rapid and life-altering decisions. Some of these decisions, such as

Corresponding Author: Patricia M. Roblin, M.S., research field: disaster preparedness. E-mail: problin@downstate.edu. those that alter standards of care, may be at odds with the Hippocratic traditions to which most physicians subscribe, particularly the requirement to do what is best for the patient without consideration of other personal or social obligations. However, they may be necessary to save a greater number of lives or to preserve civil and social order.

The ethics of rapid, often life-altering decision-making that is necessary in such circumstances should be at the forefront of disaster planning but is often left to retrospection. How does one balance the good of the larger population with that of the individual? What factors should be taken into 
account because of medical or social needs, and which ones will human nature dictate? These decisions, too frequently at the mercy of time and circumstance, have far-reaching implications that mandate genuine deliberation [4-7].

In two recent reports, the Institute of Medicine has explored ethical decision-making frameworks and discussed the logistics and implications of altering standards of care in disasters. The ethical principles discussed include fairness and equitable processes including transparency, consistency, proportionality, and accountability. These concepts have been addressed in several contexts in the disaster literature and have been applied to both actual disasters and theoretical mass casualty situations. While debate remains about the need to implement different standards of care in crises, there is a definite need to discuss the realities of these decision-making processes with physicians and first responders, patients and their families, and the community at large [8,9].

As a first step, the New York Institute for All Hazards Preparedness and the John Conley Division of Medical Ethics and Humanities at SUNY (State University of New York) Downstate Medical Center convened a working group of experts to discuss and explore the ethical decision-making that takes place during a disaster. This group was made up of experts from emergency medical services and disaster preparedness, emergency physicians and residents, clinical ethicists, and palliative care providers. This group was chosen to represent the medical, logistical, and philosophical aspects of triage with the hope of achieving a balanced and multi-faceted discussion and about confronting challenges in disaster decision-making.

The day was divided into two sessions. The morning plenary session consisted of lectures discussing disaster triage, the evolution of disaster medicine, current triage systems, and other key issues including medico-legal implications and social policies. At the end of the morning session, the audience was divided into three groups and the participants were put through a triage exercise designed to confront the dilemmas faced in actual disaster scenarios. Participants were forced to identify and address ethical concepts in decision-making, such as fairness, equitable process, consistency, proportionality, and accountability. This report provides a summary of their deliberations, explores and analyzes the key issues raised and addressed, and provides recommendations for changes in current practice and future areas for research and development.

\section{Methods}

On March 7, 2012, a symposium on Ethics in Disaster was held at the SUNY Global Center in New York City. This symposium brought together a distinguished group of experts to examine bioethics in the context of disasters. One of the aims of the symposium was to identify issues that would help to stimulate and promote further discussion of disaster bioethics. The symposium participants were a diverse group consisting of 37 professionals from the fields of emergency medicine, emergency planning, emergency services, clinical ethics, public health, palliative care and various clinical disciplines.

The participants were tasked with ethical decision-making in a disaster. This is a complex issue that is shaped by many factors that influence crisis standards of care. The symposium attendees were given a state of the art presentation followed by a plenary session, which engaged participants in discussion on current standards of care, the state of hospital response plans, and the availability of critical resources. These factors include: fairness, transparency, consistency, proportionality, accountability, public expectations and legal ramifications.

After discussing current standards of care, the state of hospital response plans, and the availability of critical resources in plenary sessions, the participants were divided into three breakout groups and presented with a detailed drill scenario involving mass casualties 
following an explosion and partial building collapse.

The groups were given additional specific information and points to remember about the event: a one-time event with national implications. In particular, the scenario involved the accidental release of a novel chemical agent (neurotoxin). The scenario presented the groups with five individual victim profiles. These victims had similar acuity and life-threatening injuries on presentation to the SUNY Downstate Medical Center and were classified as critical patients (red). There was only one trauma team available to work on the patients therefore requiring triage and prioritization. Only one patient could be treated at a time. The patients were: Captain S, an off-duty New York Police Department officer who stopped to help victims of the blast; Dr. C, a pregnant Emergency Medicine physician who was at work when the disaster occurred; Mr. M, a resident of a homeless shelter in close proximity to the explosion; Toddler B, the grandson of the Dean of the College of Medicine who was in the day care center at the disaster site; and Grandma $\mathrm{W}$ who resided in the building affected and was picking up her grandchildren while her daughter was at work in the hospital.

There was a rapid time frame for definitive care. There would be an overwhelming need for respiratory support. There would be a risk to first responders, hospital staff and the community. The hospital would be isolated from incoming resources. There would be a need for altered standards of care. Children as victims and more specifically children of medical staff needed extraordinary resources. As is widely known, medical triage at the scene and in the hospital often differ, so the participants were asked to consider the patients as they entered the Emergency Department. With that in mind, the groups would have the role of an Emergency Department provider with the duty to choose whom to treat first.

\section{Results}

\subsection{Plenary Discussion}

During the discussion preceding, the scenario-based breakout group session, participants raised the following considerations related to decision-making during a disaster:

\subsubsection{Current Standards of Care}

A standard of care may mean one thing in medical practice yet be perceived differently in law. Expressing a concern that legal liability may be a stumbling block in discussions regarding ethical decision-making in disasters, symposium participants referenced the case of Dr. Pou at Memorial Medical Center in New Orleans during Hurricane Katrina. Physicians and healthcare providers are fearful that her experience has sent a message to stay "under the radar" during such a crisis because of possible legal consequences. Although Dr. Pou's case concluded with an apology and all charges being dropped by the New Orleans District Attorney's office, there was a perception amongst discussants that modifying standards of care during disasters may potentially have civil liability and criminal justice implications and that these must be examined and considered.

\subsubsection{State of Hospital Response Plans}

The public expectation is that healthcare providers can do everything for everyone. When this is not possible, it will be important to explain the limitations of certain situations to them. To maintain public confidence during disasters, transparency is critically important and the public needs to be provided with an honest appraisal of what is happening. In one example, participants stressed that in order for hospital triage policy to change, the public must become involved in the discussion. Only by making the public a stakeholder in the decisions made by the hospital will the public expectation of providers reflect the reality of the resources available.

Participants also felt that any hospital triage policy must adhere to a national standard. However, it was noted that a policy for New York City could vary significantly from other jurisdictions given the uniqueness of the NYC system and health care delivery within the five boroughs. One thought was that it was 
feasible to build national guidelines for hospital triage, which local communities could use to write their individual plans. Coincidentally, this concept was not supported by the March 2012 release of CDC's Public Health Preparedness Capabilities: National Standards for State and Local Planning in which the States are called upon to provide guidance and protocols on crisis standards of care "in order to enable a substantial change in routine healthcare operations including the delivery of the optimal level of patient care for a pervasive ... disaster."

The IOM's (Institute of Medicine) 2009 Guidance for Establishing Crisis Standards of Care for Use in Disaster Situations and its five key areas that would serve as a framework for the creation of those standards was also discussed by participants. While the 2009 report did not provide a framework in which to create standards, some participants alluded to the release of the March 21, 2011 IOM report and they hope that this document would provide specific steps to create a comprehensive crisis standards framework for communities.

\subsubsection{Availability of Clinical Resources}

Management of scarce resources was identified by participants as a function within surge planning-federal target capability. In fact, providing guidance for the management of scarce resources is a planning element of Medical Surge (Capability 10, Function 4) in the above CDC publication. Many of the process-related strategies for managing scarce resources that were discussed during the symposium are outlined in the $\mathrm{CDC}$ document.

3.1.4 Additional Questions Posed to the Symposium Participants Included:

- Is it possible to make Regional Plans?

- Does the public understand "scarcity of resources"?

-Will a plan pigeon-hole or liberate care providers?

- Is this a Public Relations issue?

\subsection{Breakout Groups Discussion}

The main themes that were discussed during the breakout groups' discussion are summarized for all groups. Suggestions that were common amongst participants as well as significant differences in proposed solutions are mentioned below.

3.2.1 Priority of Treatment and Ethical Considerations

The BG (breakout groups) Considered various approaches to prioritizing patients within the disaster scenario. The following issues were considered:

- Patient age and overall health (co-morbidities);

- Prognosis based on clinical symptoms, severity of exposure, and expectation for survival including co-morbidities;

- Patients value to society and their relationship to care providers;

- Community acceptance and perceptions of physician actions;

- Egalitarian approach.

Although the patient scenarios were purposely constructed to have similar levels of acuity and prognosis, all three breakout groups initially tried to triage the patients according to their clinical presentations and co-morbidities. Military and emergency medical services triage protocols were initially used, although similarities in presentations made it difficult to decide treatment solely on these models. Additionally, although there was a desire to incorporate medical co-morbidities into the decision making process, it was quickly determined to be an impractical consideration because the information would be unlikely to be immediately available and might be falsified in an effort to encourage treatment. There was also controversy about whether the EMS (emergency medical services) triage model would lead to either none of the patients to be transported to the hospital (black tagged) or all of them (scoop and run method).

Groups discussed treating patients on a first-come, first served basis to help allow for equality and alleviate moral anguish. However, this approach was dismissed by some on the basis that it was irrelevant 
because many patients would arrive at approximately the same time. Many participants also felt that it was not a practical approach and contrary to emergency practice, since that policy may lead to treating patients who cannot be saved over those that could or not addressing the most dire emergencies first.

The controversial concept of "social value" was also discussed, primarily whether it has an impact on practitioner decision-making and if it would affect treatment decisions on either a conscious or sub-conscious level. Most of the participants tried to leave this consideration aside when discussing Mister $\mathrm{M}$, the homeless patient, but became acutely aware of its importance in decision making when discussing the doctor and police captain. Although the groups agreed that social value should not be overtly used in the decision making process, most admitted to having an inherent bias that would lead them to care for the uniformed officer or physician before a homeless man or someone with an extensive drug or alcohol use history. Additionally, there was a concern that first responders may not volunteer to help in disaster scenarios in the future if they did not believe that their care would be prioritized if they were injured. Several participants from EMS backgrounds openly stated that their relationship with other uniformed members was akin to a family, and that they and others in their services would treat and transport their own members over unaffiliated patients.

Ultimately, the groups triaged the patients based primarily on their clinical presentations and likely prognosis, and then referring to age and professional affiliations in the case of similar medical conditions. Table 1 shows the results of the triage, separated by group.

Table 1 Team triage order.

\begin{tabular}{llll}
\hline & Blue & Orange & Yellow \\
\hline 1 & Doctor C & Toddler B & Doctor C \\
2 & Toddler B & Doctor C & Toddler B \\
3 & Mister M & Captain S & Captain S \\
4 & Captain S & Mister M & Mister M \\
5 & Grandma W & Grandma W & Grandma W \\
\hline
\end{tabular}

Overall, the three groups ultimately had a very similar rationale in making their ranking orders. The blue and yellow groups triaged Dr. C first on the basis that her condition was not due to the toxin but rather because of trauma, that her blood loss was potentially reversible, and that saving her would do the most good because two live - hers and the unborn child's - would actually be saved. However, there was a concern that it might be unrealistic to expect adequate care for the newborn as the skill set of the care providers was unknown. Toddler B would be the next because he is the youngest with the longest potential life span and best possible quality of life if he survives. The orange group ranked the toddler first and the doctor second because they interpreted the toddler's respiratory symptoms as the most reversible and therefore meriting immediate action. Captain $\mathrm{S}$ was ranked third by two groups, balancing the severity of her clinical presentation with her uniformed status. The blue group, however, triaged the captain fourth, basing their decision entirely on clinical presentation and consciously deciding not to weight her social utility against Mister M's. All of the groups agreed that Mister M would be treated before the grandmother despite a lesser perception of his usefulness to society because his condition was not as severe and potentially reversible with rapid treatment. There was also consensus that Grandma W should be triaged last with the opinion that her age, likely co-morbidities, and extensive trauma would preclude a good outcome and that the resources required to attempt treatment could not be justified in this context. The groups agreed that Mister $\mathrm{M}$ would be treated before the grandmother despite a lesser perception of his usefulness to society because his condition was not as severe and potentially reversible with rapid treatment.

Overall, despite all of the groups having a lengthy initial discussion about ethical and societal considerations and the desire to appear neutral, patients clinical presentation was followed closely by age, usefulness to society and identity as a uniformed or 
clinical care provider as the most important considerations.

\subsubsection{Leadership and Logistics}

One of the major recommendations to arise from this conference was the importance of strong leadership during a disaster. The groups recommended that a senior physician be assigned as a primary decision maker and leader in a mass casualty incident. This individual would be the most senior person available on the scene when the incident occurs. They would not be directly involved in patient care during the incident but would instead direct and allocate resources and apply pre-determined criteria when making treatment decisions.

\subsubsection{Training and Preparedness}

Along those lines, it was recommended that institutions identify a group of senior personnel to receive special training on an ongoing basis in order to be prepared to take on this leadership role in the case of a mass casualty incident. This training should be focused on disaster preparedness, triage principles, and bioethics in order to facilitate the best possible decisions. Although it was strongly felt that the "in charge" individual should usually be a physician, it was agreed that relevant training should be provided for an interdisciplinary group including ethicists, administrators, chaplains, palliative care specialist and community representatives alongside physicians.

\subsubsection{Community Involvement and Outreach}

Community perception of the handling of a disaster and their biases in determining treatment was another major factor that influenced decision-making. Overall, the breakout groups believed that Americans have a sense of abundance and entitlement to the best care all of the time and that, even in a disaster scenario, communities would assume that resources are plentiful and that all patients could be saved. Although equipment, medications, and providers are, in reality, limited resources, it was felt that this information has not been adequately shared with the community. There was concern that following such a disaster, there would be decreased trust towards the providers and that riots or other public protest could ensue, potentially distracting care and endangering even more people.

The BG all agreed that communication and engagement of the community prior to a disaster was the ideal. By laying out clear information about resources, disaster plans and possibly engaging the community more extensively in drills, citizens may have clearer and more realistic expectations during a disaster. Clergy may play a special role in supporting patients and families, who cannot receive active therapeutic interventions due to futility or unavailable resources. Interested community members might also receive training in order to make them useful volunteers in a disaster. Protocols were also recommended to help practitioners keep emotion out of the care plan.

\subsubsection{Palliative Care}

The BG also agreed that the availability of a PC (palliative care) team would be of critical assistance to the medical care providers. The PC team would help expand care options and help families navigate through care plans. There is also a need to identify appropriate palliative care options during a disaster and to identify whether they require professionals to administer them or whether there is a role for ancillary personnel or volunteers. Use of non-standard practitioners maybe an untapped resource that needs to be considered. This group might include: medical students, PA students, other hospital staff, off-duty EMS personnel, and the previously mentioned community individuals who may have basic skills to assist in bagging intubated patients, transporting patients etc. or who may be able to provide emotional support to patients and families. The development of protocols and educational initiatives for such individuals was recommended.

Post incident stress debriefing should be part of any disaster. Though no clear method was found to be superior, the BG felt this was an important and necessary element. It should also continue over time. 
This would make participation in a future incident more likely.

\section{Key Recommendations}

There is a need for clear, easy-to-follow, transparent and equitable crisis standards of care that will support making rational decisions that may run counter to usual expectations of patient care providers.

During a mass casualty incident, one individual, preferably an experienced clinician, who is removed from direct patient care, with prior bioethics and leadership training, should make treatment decisions.

Training and drills need to exercise the leadership role of the person in charge of making ethical treatments decisions during an overwhelming event to ensure that their respective organizations support this function.

Participation of a palliative care teams during crisis and disaster situations would expand the options of care and help the family feel more comfortable with difficult decisions regarding limiting care for their loved ones.

The roles of community members (such as but not limited to clergy) during disasters should be defined and ongoing training at hospitals should include the community at large

Community involvement prior to catastrophic events is critical so there is an understanding of the basis for ethical decision-making about medical care and participation in the planning process.

Mental health support including post-incident stress debriefing for hospital staff should be part of protocols and the planning for disasters as staff may be experiencing trauma from withholding care.

Maximizing resource re-allocation should be included in the discussion so that opportunities for recruiting additional support are not missed (e.g., credentialing EMS personnel to assist with certain functions in hospitals).

\section{Acknowledgements}

This publication was supported by the John Conley Division of Medical Ethics and Humanities, SUNY Downstate Medical Center.

\section{References}

[1] Merin, O., Ash, N., Levy, G., Schwaber, M. J., and Kreiss, Y. 2010. "The Israeli Field Hospital in Haiti-Ethical Dilemmas in Early Disaster Response." N. Engl. J. Med. 362 (11): e38.

[2] Okie, S. 2008. "Dr. Pou and the Hurricane-Implications for Patient Care during Disasters" N. Engl. J. Med. 358 (1): $1-5$.

[3] McKenna, M. 2011. "Reaping the Whirlwind: Emergency Physician Recounts His Fateful Brush with Deadly Twister in Joplin, MO.” Ann. Emerg. Med. 58 (3): A17-9.

[4] Institute of Medicine. 2009. Guidance for Establishing Crisis Standards of Care for Use in Disaster Situations: A Letter Report. Washington, DC: National Academies Press.

[5] Institute of Medicine. 2012. Crisis Standards of Care: A Systems Framework for Catastrophic Disaster Response. Washington, DC: National Academies Press.

[6] Coleman, C. N., Knebel, A. R., Hick, J. L., Weinstock, D. M., Casagrande, R., Caro, J. J., DeRenzo, E. G., Dodgen, D., Norwood, A. E., Sherman, S. E., Cliffer, K. D., McNally, R., Bader, J. L., and Murrain-Hill, P. 2011. "Scarce Resources for Nuclear Detonation: Project Overview and Challenges." Disaster Med. Public Health Prep. 5 (Suppl 1): S13-9.

[7] Caro, J. J., DeRenzo, E. G., Coleman, C. N., Weinstock, D. M., and Knebel, A. R. 2011. "Resource Allocation after a Nuclear Detonation Incident: Unaltered Standards of Ethical Decision Making." Disaster Med. Public Health Prep. 5 (Suppl 1): S46-53.

[8] Hick, J. L., Hanfling, D., and Cantrill, S. 2012. "Allocating Scarce Resources in Disasters: Emergency Department Principles" Ann. Emerg. Med. 59 (3): 177-86.

[9] Schultz, C. H., and Annas, G. J. 2012. "Altering the Standard of Care in Disasters-Unnecessary and Dangerous.” Ann. Emerg. Med. 59 (3): 191-5. 